\title{
CME dynamics using coronagraph and interplanetary ejecta observations
}

Alisson Dal Lago, Walter D. Gonzalez, Carlos R. Braga, Aline de Lucas, Lucas R. Vieira and Tardelli R. C. Stekel National Institute for Space Research, INPE, Space Geophysics Division, São José dos Campos, SP, Brazil

Copyright 2011, SBGf - Sociedade Brasileira de Geofísica

This paper was prepared for presentation during the $12^{\text {th }}$ International Congress of the Brazilian Geophysical Society held in Rio de Janeiro, Brazil, August 15-18, 2011.

Contents of this paper were reviewed by the Technical Committee of the $12^{\text {th }}$ International Congress of the Brazilian Geophysical Society and do not necessarily represent any position of the SBGf, its officers or members. Electronic reproduction or storage of any part of this paper for commercial purposes without the written consent of the Brazilian Geophysical Society is prohibited.

\section{Abstract}

In this work, we present a study of coronal mass ejection (CME) dynamics using LASCO coronagraph observations combined with in-situ ACE plasma and magnetic field data, covering a continuous period of time from Jan. 1997 to April 2001, complemented by few extreme events observed in 2001 and 2003. We find that the CME expansion speed correlates fairly well with the travel time to $1 \mathrm{AU}$ of the interplanetary ejecta (or ICMEs) associated with the CMEs, as well as with their preceding shocks. The dataset used in this work is a subset of the one from Schwenn et al. (2005), from which only the CMEs associated with interplanetary ejecta (ICMEs) were selected. Three models to predict $\mathrm{CME}$ travel time to Earth, two proposed by Gopalswamy et al. (2001) and one by Schwenn et al. (2005), were used to characterize the dynamical behavior of this set of events. Extreme events occurred in 2001 and 2003 were used to test the prediction of the models to a larger range of $\mathrm{CME}$ energies.

\section{Introduction}

One of the key issues of Space Weather is the dynamics of coronal mass ejections (CMEs), from their release from the Sun to their propagation throughout the interplanetary (IP) space, eventually impacting the Earth and other planets. These impacts of CMEs are the most important drivers of space weather phenomena (Gonzalez et al. 1999).

A number of empirical and analytical studies have addressed this point so far, using observations from coronagraphs and interplanetary monitors, in order to correlate CMEs observed near the Sun and in-situ, e.g. Earth vicinity (Gopalswamy et al. 2001, Schwenn et al. 2005). However, error bars in CME travel time predictions from the Sun to the Earth are of the order of \pm 1 day, which is considerably large for the typical time scale of 1 to 3 days of their travel time. After many years of intensive investigation of CMEs observed with the Large Angle and Spectrometric Coronagraph (LASCO), aboard the Solar and Heliospheric Observatory (SOHO), we found that the subset of interplanetary counterparts of CMEs, the ICMEs, with a well defined ejecta structure are those with the best predictable behavior. The relationship between CME expansion speed and interplanetary ejecta travel time to Earth for these events, using coronagraph observations, is the one with the lowest scatter among other sets of events, such as interplanetary shocks.

This work presents a statistical study of all the CMEejecta and shock events observed by $\mathrm{SOHO}$ and by the Advanced Composition Explorer (ACE) satellite during the continuous time interval from Jan. 1997 to April 2001, complemented by few extreme events occurred in 2001 and 2003.

\section{Method}

Observations used in this work are from two distinct sources: one observing remotely the vicinity of the Sun; and the other observing near Earth in-situ solar wind plasma and magnetic field, covering a continuous time interval from January 1997 to April 2001, and some few observations of extreme events in 2001 and 2003.

The Large Angle and Spectrometric Coronagraph (LASCO) (Brueckner et al., 1995), onboard the Solar and Heliospheric Observatory (SOHO) (Howard et al., 1997) is able to observe the plane-of-sky solar corona in white light from 2 to 32 solar radii. Sequences of LASCO images can reveal the plane-of-sky propagation of CMEs.

Earth-directed CMEs are expected to have large apparent angular size, named halos (Howard et al., 1982), which can be partial or full. Complementary to LASCO CME observations, the Extreme Ultraviolet Imaging Telescope (SOHO/EIT) provides observations of the solar disk CME related activity in UV wavelength, which allows the identification of CMEs as front side (ejected towards the observer) or back side (ejected away from the observer).

Near Earth in-situ plasma and magnetic field observations are continuously provided by the instruments onboard the Advanced Composition Explorer (ACE) satellite, located in the L1 Lagrangean point. Although unidimensional, these observations enable the identification of interplanetary structures, such as shocks, sheaths and interplanetary ejecta structures related to CMEs (Neugebauer and Goldstein, 1997).

Of special importance is the correct association of structures present in both datasets, i. e., the association between near Sun and near Earth phenomena. 
The higher the solar activity, the more difficult this correlation becomes, because the daily CME rate varies from 0.2 to 5 , in solar minimum and solar maximum period, respectively (St. Cyr et al., 2000). Their typical propagation time from the Sun to the Earth is from 1 to 3 days (Schwenn et al., 2005).

In this work, association was made using careful inspection of each single event, taking into account the whole dataset available for this period. For a given LASCO CME, a time window of 1 to 3 days was established, in order to look for interplanetary (IP) signatures. If a single IP event was identified inside the time window, this was considered to be associated with the given LASCO CME. If more than one CME and IP signatures were indentified inside a time window, correspondence was assumed to be chronological (first CME correlates to the first IP event, second CME correlates to the second IP event, and so on). This same dataset was used in previous works (Dal Lago et al. 2004, Schwenn et al. 2005). In addition to this dataset, we selected few other extreme events occurred in 2001 and 2003, which were not included in the first dataset. These events are very important to evaluate the behavior of CMEs at higher energetic conditions.

The halo CME expansion speed is the growth rate of the CME, perpendicular to its largest plane-of-sky speed direction (Dal Lago et al. 2003). The CME expansion speed was shown to be very well-correlated to the CME radial speed (Dal Lago et al., 2003) and it was used to study IP shock propagation from the Sun the the Earth (Schwenn et al., 2005).

We used some of the criteria of Neugebauer and Goldstein (1997) to identify IP ejecta associated with solar CMEs, the ICMEs: high magnetic field magnitude with low variance, in some cases smooth magnetic field rotation (magnetic clouds), low temperature, and low plasma beta.

\section{Example}

Figure 1 shows the interplanetary ejecta observed in the Earth's vicinity by ACE satellite in August 2000. From top to bottom, the Figure presents the total intensity of the interplanetary magnetic field (B), its $z, y$ and $x$ components $(B z, B y$ and $B x)$, solar wind velocity $(V)$, number density $(\mathrm{N})$ and temperature $(\mathrm{T})$, and the geomagnetic Disturbance storm-time Dst index.

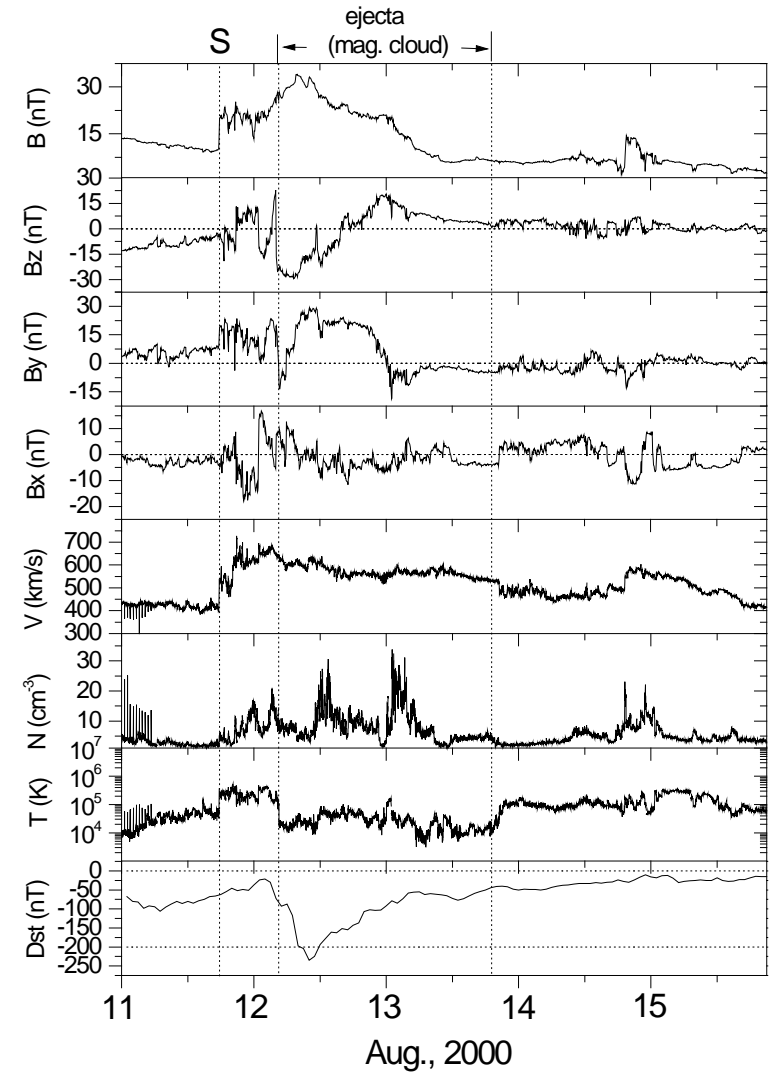

Fig. 1. Interplanetary ejecta observed in the Earth vicinity by ACE satellite in August 2000. From top to bottom, the Figure presents the total intensity of the interplanetary magnetic field $(B)$, its $z, y$ and $x$ components (Bz, By and $B x)$, solar wind velocity $(V)$, number density $(\mathrm{N})$ and temperature $(\mathrm{T})$, and the geomagnetic Disturbance storm-time Dst index.

An IP shock (S) is identified by the abrupt jump in all parameters in August 11th, at 18:19 UT. On August 12th, at 06:00 UT, the total magnetic field becomes smoother and the temperature drops, indicating that ACE was crossing an interplanetary ejecta. A smooth magnetic field rotation is also observed inside this ejecta, indicating that this ejecta is consistent with Burlaga et al. (1981) definition of a magnetic cloud. A geomagnetic storm was observed as a consequence of the passage of this ICME through the Earth, as indicated by the drop of the Dst index (bottom panel of Figure 1).

\section{Results}

Gopalswamy et al. (2001) proposed models for CME travel time to $1 \mathrm{AU}$ using empirical relations (linear and quadratic) between the CME initial speed measured in LASCO coronagraph and the average CME-ICME acceleration, using observations close to the Sun and in the interplanetary space. In their models, acceleration was considered to cease at $0.76 \mathrm{AU}$. The authors used as CME initial speed the plane-of-sky speed of the fastest 
CME moving feature (for further details on these models, please refer to Gopalswamy et al. 2001).

In order to evaluate this model with our dataset, an adaptation was made, using CME expansion speed (Vexp) instead of CME plane-of-sky speed. Empirical relations between Vexp and CME average acceleration were calculated. The outputs of the models (both using linear and quadratic acceleration) are shown in Figure 2, lines 1 and 2, respectively. Also shown in the Figure are the data points from the period from Jan. 1997 to April 2001, and the extreme events observed in 2001 and 2003. It must be pointed out that these extreme events from 2001 and 2003 were not used to produce the model parameters.

Using the same methodology as Schwenn et al. (2005), a model of CME travel time to $1 \mathrm{AU}$ was used to fit the continuous period dataset in Figure 2 (only the data from Jan. 1997 to April 2001, excluding the 2001 and 2003 extreme events). This model is shown as curve 3 in Figure 2, and the equation is given by:

$t=263-28.28 \ln (V e x p)$

where $\mathrm{t}$ is the CME travel time from the Sun to $1 \mathrm{AU}$, given in hours, and Vexp is the CME expansion speed, given in $\mathrm{km} / \mathrm{s}$.

Additional to these model curves in Figure 2, we present a constant speed radial propagation (kinematic approach) in curve 4 of Figure 2.

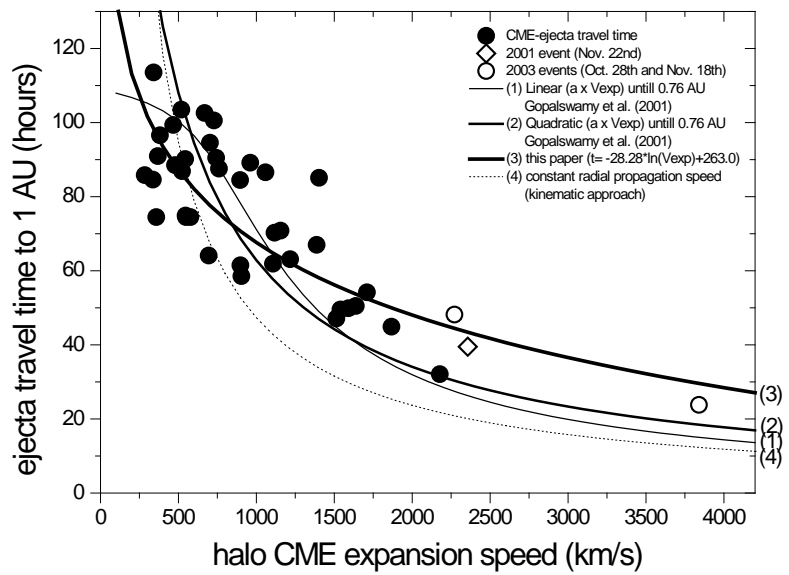

Figure 2. Ejecta travel time to $1 \mathrm{AU}$ versus the CME expansion speed for 38 events observed in a continuous period from Jan. 1997 to April 2001 (filled circles) and some extreme events observed in 2001 (open diamond) and 2003 (open circles). Prediction models from Gopalswamy et al. (2001) (lines 1 and 2), this work (after Schwenn et al., 2005) (curve 3) and kinematic approach (line 4) are shown.

It is interesting to note that nearly all models represent the behavior of the dynamics of the CMEs fairly well. The model based on Schwenn et al. (2005) methodology is slightly better, specially for the 2001 and 2003 extreme events.

Figure 3 shows the shocks associated with the CMEs of Figure 2. Note that there are few additional extreme events in this Figure, which did not have IP ejecta associated with them (thus, they are not present in Figure 2). The models shown in the Figure represent basically the same ideas of Figure 2, except for the fact that the IP phenomena considered here are IP shock arrivals, instead of ejecta arrivals in Figure 2. Compared to the ejecta analysis, models for shock arrival seem to agree more, specially for the extreme events.

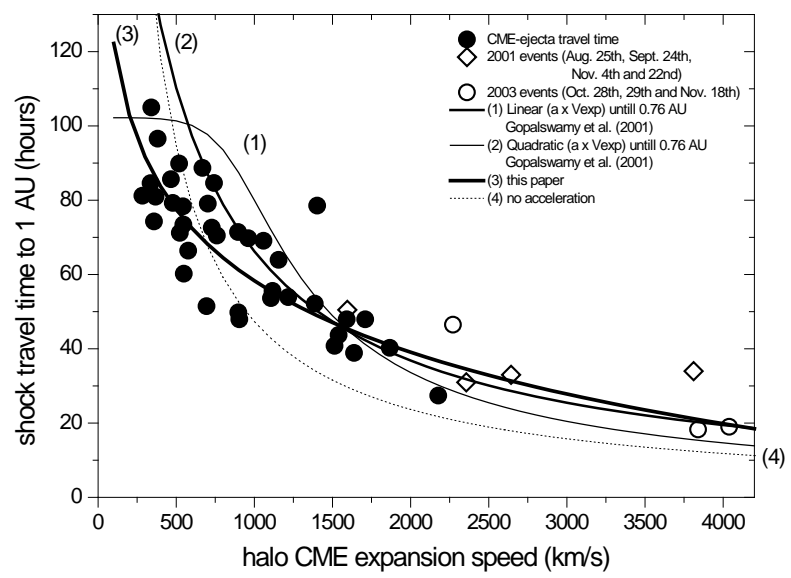

Figure 3. Shock travel time to $1 \mathrm{AU}$ versus the CME expansion speed for 38 events observed in a continuous period from Jan. 1997 to April 2001 (filled circles) and some extreme events observed in 2001 (open diamond) and 2003 (open circles). Prediction models from Gopalswamy et al. (2001) (lines 1 and 2), this work (after Schwenn et al., 2005) (curve 3) and kinematic approach (line 4) are shown.

\section{Conclusions}

In this work we presented a study of the dynamics of CMEs using observations from the Large Angle and Spectrometric Coronagraph, combine with in-situ observations from the Advanced Composition Explorer (ACE) satellite. Data used in this work was a subset of Schwenn et al. (2005) dataset, from which only the CMEs associated with IP ejecta were taken into account. From the analysis, it was found that this dataset of CME-ejecta events is much better described by CME travel time models than the entire collection of CME-shock events. Empirical models proposed by Gopalswamy et al. (2001) and Schwenn et al. (2005) were able to describe fairly well the behavior of extreme events occurred in 2001 and 2003, which were not used to calculate the model parameters, with little better performance of the latter for the case of CME-ejecta events. 


\section{Acknowledgments}

Authors would like to thanks for support granted to this research: A. Dal Lago - CNPq (481368/2010-8 and 303798/2008-4); C. R. Braga - CAPES; A. de Lucas FAPESP (2008/10791-7); L. R. Vieira - FAPESP (2009/12869-6), T. R. C. Stekel - FAPESP (2010/137077). The authors would like to acknowledge the LASCO and EIT team for the solar data used in this work. We would also like to acknowledge N. Ness (Bartol Research Institute), D. J. McComas (Southwest Research Institute), R. Lepping (NASA/GSFC), K. Ogilvie (NASA GSFC) and CDAWeb for interplanetary magnetic field and plasma data. SOHO is a project of international cooperation between ESA and NASA.

\section{References}

Brueckner, G. E., Howard, R. A., Koomen, M. J., Korendyke, C. M., Michels, D. J., Moses, J. D., Socker,D. G.,Dere, K. P.,Lamy, P. L.,Llebaria, A.,Bout, M.V., Simnett,G.M., Bedford,D. K., and Eyles, C. J.: 1995, Solar Phys. 162, 357.

Burlaga, L. F.; Sittler, E.; Mariani, F.; e Schwenn, R. Magnetic loop behind an interplanetary shock: Voyager, Helios and IMP-8 observations. J. Geophys. Res., v.86, n.A8, p.6673-6684, Aug. 1981.

Dal Lago, A., Schwenn, R., and Gonzalez,W. D.: Relation between the radial speed and the expansion speed of coronal mass ejections, Adv. Space Res., 32, 26372640, 2003

Dal Lago, A., Vieira, L. E. A. Echer, E., Gonzalez, W. D., Clúa de Gonzalez, A. L., Guarnieri, F. L., Schuch, N. J. Schwenn, R., Comparison between halo CME expansion speeds observed on the sun, the related shock average propagation speeds to earth and corresponding ejecta speeds at $1 \mathrm{AU}$. Solar Physics 222: 323-328, 2004.

Gonzalez, W.D., B.T. Tsurutani, e A.L.C. De Gonzalez, Interplanetary origin of geomagnetic storms, Space Science Reviews, 88 (3-4), 529-562, 1999.

Gopalswamy, N. A. Lara, S. Yashiro, M. L. Kaiser, and R. A. Howard, Predicting the 1-AU arrival times of coronal mass ejections, J. Geophys. Res., 106, A12, 2920729217, 2001

Howard, R. A., Michels, D. J., Sheeley Jr., N. R., and Koomen, M. J.: 1982, Astrophys. J. 263, 101.

Howard, R. A., Brueckner, G. E., St. Cyr, O. C. Biesecker, D. A., Dere, K. P., Koomen, M. J., Korendyke, C. M., Lamy, P. L., Llebaria, A., Bout,M. V., Michels, D. J.,Moses, J. D., Paswaters, S. E., Plunkett, S. P., Schwenn, R., Simnett, G. M., Socker, D. G., Tappin, S. J., and Wang, D.: 1997, in N. Crooker, J. A. Joselyn, and J. Feynman (eds.), 'Observations of CME from SOHO/LASCO', Coronal Mass Ejections, Washington, DC, AGU, pp. 17-26.

Neugebauer, M. and Goldstein, R.: 1997, Particle and Field Signatures of Coronal Mass Ejections in the Solar Wind. in N. Crooker, J. A. Joselyn, and J. Feynman (eds.), Coronal Mass Ejections, Washington, DC, AGU, pp. 245-251.
Schwenn, R., Dal Lago, A., Gonzalez, W. D., Huttunen, E., The association of coronal mass ejections with their effects near the Earth,Ann. Geophysicae, 23, 10331059, 2005.

St. Cyr, O. C., Howard, R. A., Sheeley Jr., N. R., Plunkett, S. P., Michels, D. J., Paswaters, S. E., Koomen, M. J., Simnett, G. M., Thompson, B. J., Gurman, J. B., Schwenn, R., Webb, D. F., Hildner, E., and Lamy, P. L.: 2000, J. Geophys. Res. 105, 18169. 